\title{
Plate-Separation Requirements for Standard Free-Air Ionization Chambers
}

\author{
Frank H. Attix and LeRoy DeLaVergne
}

\begin{abstract}
A method is described for measuring ionization losses resulting from inadequate separation of the parallel plates in free-air chambers. The ionization produced by X-rays scattered out of the collimated beam was measured separately and subtracted from the total to obtain the ionization contribution of only the electrons from the beam. Losses of this electron ionization are tabulated for plate separations from 6 to 36 centimeters, with moderately and heavily filtered X-rays from 50- to 250-kilovolt constant tube potential. Results are also converted into cylindrical coordinate form for application to chambers other than the parallel-plate type. A method is suggested for measurement, in other free-air chambers, of the contribution of ionization by scattered X-rays. Ionization-loss results are compared with previous experiments.
\end{abstract}

\section{Introduction}

For many years the free-air ionization chamber has been the standard instrument for the measurement in roentgens of X-radiation dose below 200 kilovolts. Agreement between the standard chambers of several national laboratories within about \pm 1 percent was established in 1931 by Taylor $[1,2],{ }^{2}$ and the design of the standard chamber $[3,4]$ at the National Bureau of Standards has remained unchanged from that time until recently.

In 1952, Kemp and Hall [5] made a precise study of the lateral dimension requirements for complete utilization of electron ionization in a parallel-plate, variable-pressure air chamber. The results indicated that the standard chambers at the National Bureau of Standards and at the National Physical Laboratory in England were inadequate in this respect, and that electric field distortion was responsible for the consistant underestimation of plateseparation requirements by other workers.

Preliminary measurements at the Bureau early in 1953, using a large free-air chamber with improved distortion characteristics, indicated that even larger chamber dimensions might be required than those predicted by Kemp and Hall. On the basis of this preliminary work, a temporary standard was constructed having plates $20 \mathrm{~cm}$ in separation and 26.8 $\mathrm{cm}$ in height. A comparison between this chamber and the old NBS standard chamber with X-rays of $200 \mathrm{kv}$ (constant potential) and medium filtration $(0.52-\mathrm{mm} \mathrm{Cu}$ plus $1.0-\mathrm{mm} \mathrm{Al})$ revealed that the old chamber was measuring about 2 percent less ionization than the new one, in fair agreement with the predictions of Kemp and Hall.

A direct intercomparison between the National Physical Laboratory chamber and the new National Bureau of Standards chamber was carried out at NPL in June of 1953 [6]. The NPL chamber was found to be subject to ionization losses of about 3 percent at $195 \mathrm{kv}$ (constant potential), which was

\footnotetext{
1 This work was supported in part by the Atomic Energy Commission. This paper was presented at the December 16,1953 , meeting of the Radiological Society of North America, and appears in the December 1954 issue of

Radiology.
Figures in brackets indicate the literature references at the end of this paper.
}

close to the results Kemp and Hall obtained with a replica of the NPL chamber.

However, because of the disagreement between their work and the preliminary measurements made at the Bureau, and because their evidence placed in doubt the previously accepted free-air chamber design criteria, a broad study of the problem was undertaken. Both the field-distortion and plateseparation characteristics of free-air chambers are being investigated at the Bureau for X-rays generated by potentials up to $500 \mathrm{kv}$. This present paper deals primarily with the plate-separation requirements for X-rays generated by potentials up to $250 \mathrm{kv}$.

The definition of the roentgen requires the measurement of all the ionization produced by electrons that originate within a known volume of irradiated air. It is not practicable to measure this ionization however, because one cannot separate it from the ionization produced by other electrons originating in neighboring volumes of air, which must, of necessity, also be irradiated. The free-air chamber overcomes this difficulty by utilizing the "electronic-equilibrium" condition existing along the direction of the $\mathrm{X}$-ray beam, as shown in figure 1 . The cross-hatched region represents the known volume in the sense of the roentgen definition. Its dimensions are established by the area of the limiting diaphragm, D, and the length of ion-collecting plate $\mathrm{C}$ (assuming a uniform electric field). Ionization is collected throughout the region enclosed by the dashed lines. Some electrons, such as $e_{1}$, having paths predominantly perpendicular to the X-ray beam will expend their entire energies within the ion-collecting region, assuming the plate separation to be sufficiently large. (Actually, of course, the electron paths are not straight as indicated.) Others, like $e_{2}$, will pass out of that region and produce ionization where it will be collected on one of the guard plates and, hence will not be measured. Under the electronic-equilibrium condition, this ionization loss is just compensated by the contribution of other electrons, such as $e_{3}$, assuming air attenuation of the primary beam to be small. Such a condition can only exist if the two ends of the box are at a distance (from the ion-collecting region) 


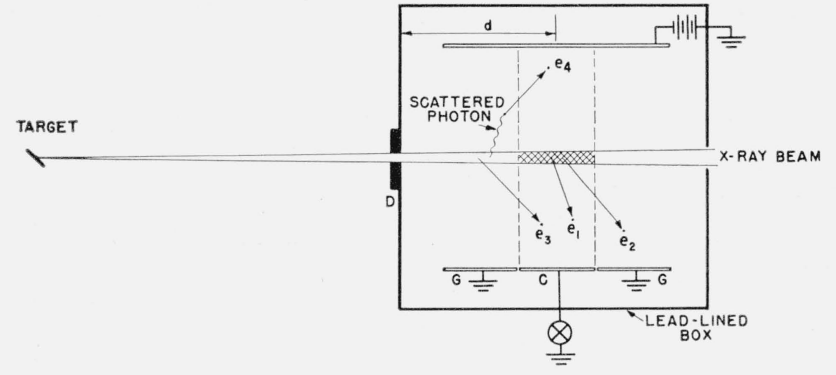

Figure 1. Schematic plan view of a parallel-plate free-air ionization chamber.

greater than the range of the electrons present, or about half the distance required for plate separation.

Electrons like $e_{4}$, resulting from scattered photons, produce ionization that is not included in the definition of the roentgen as these electrons do not originate within the cross-hatched volume of air nor do they compensate for ionization losses of other electrons that do. Their ionization contribution is usually assumed to be negligible. However, in large chambers that assumption may not be justified. On the other hand, the presence of such unwanted ionization would tend to compensate for loss of electron ionization (i. e., ionization produced by electrons that originate within the X-ray beam) due to insufficient plate separation. A method will be described for measurement of this ionization by scattered X-rays in free-air chambers.

Some mention should be made of the correction for air attenuation of X-rays in the distance $d$ (see fig. 1). Because limiting diaphragm D determines the total X-ray flux passing into the chamber, a free-air chamber measurement actually establishes the dose at the position of that diaphragm, except for the small attenuation of the beam in traversing the air between the diaphragm and the ion-collecting region. ${ }^{3}$

\section{Apparatus and Experiment}

\subsection{General Method}

The experiment consisted of three types of measurements for each kilovoltage and filtration:

1. Determination of the ionization produced in parallel layers of air, each $1 \mathrm{~cm}$ in thickness, at various perpendicular distances from a narrowly collimated X-ray beam, using a special experimental "grid" chamber.

2. A repeat of 1 , except that a sheet of material (hereafter called the "electron filter") was interposed

\footnotetext{
3 The effective length $d$, of the absorbing air column is subject to some uncertainty because it depends on the average center of origin of the electrons contributing to the ionization in the collecting region. Taking this position at the center of the collecting region assumes that electron ranges are short, or that their paths are homogeneous in direction forward and rearward. At higher X-ray energies whereforward-directed electrons predominate, the center of origin shifts toward the diaphragm and the effective air-absorption column decreases in length. If the scattered-photon ionization in a chamber is measured and corrected for in the dose calculation, then to be consistent the total absorption coefficient should be used in the air-absorption correction; that is, the coefficient should be experimentally determined by a method involving "narrow-beam" geometry, where the variable air column lies outside the narrowly diaphragmed chamber box, rather than by moving the plate system within the box.
}

between the X-ray beam and the ion-collecting region to absorb all the electrons originating in the beam, but allow scattered X-rays to pass through with little attenuation, making it possible to differentiate between the two. By subtracting the ionization measured with the electron filter in place from that measured without the filter, the ionization was obtained for only the electrons originating in the beam, as required by the roentgen definition.

3. Measurement of the ionization produced in a conventional free-air chamber by the same X-ray beam used in the first two types of measurements. This was done to provide a figure for the ionization integrated over the entire ionized volume, so that the previous measurements of ionization in various parts of the volume could be expressed as fractions to the whole.

From these measurements it was possible of calculate the percentage by which the ionization produced by electrons from the X-ray beam in a free-air chamber would be decreased by inadequate separation of the parallel plates.

\subsection{Grid Chamber}

Figure 2 is a plan view of the experimental ionization chamber. It was housed within a lead-lined box, A, $70 \mathrm{~cm}$ long by $33 \mathrm{~cm}$ wide by $39 \mathrm{~cm}$ high. The electrode system was positioned along one side wall of the box, and consisted of a graphite plate, $K$, for collecting the ionization, surrounded by coplanar guard plates, J. In front of the collector were two parallel grids of graphite-coated nylon thread, L, which defined the volume in which ionization was collected (shaded area in fig. 2). The first row of threads was $1 \mathrm{~cm}$ from the collector plate, and was operated at $\pm 135 \mathrm{v}$, which was found to be ample to avoid ion recombination in the collecting volume. The second row was at ground potential to give a more clearly defined collecting volume by reducing the "bulging" of field lines between the threads of the first row.

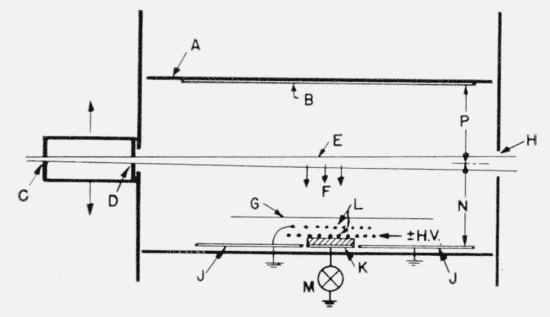

Figure 2. Plan view of grid chamber.

A, Lead box $70 \mathrm{~cm}$ long, $33 \mathrm{~cm}$ wide, $39 \mathrm{~cm}$ high; B, 1/16 in. Lucite lining, graphite-coated; C, defining diaphragm, 1-cm diam, $76 \mathrm{~cm}$ from tube target; D, scatter diaphragm, 1.8-cm diam, $17 \mathrm{~cm}$ from defining diaphragm; E, collimated X-ray beam, approx 1.8 $\mathrm{cm}$ diam at position opposite collecting electrode; F electrons position opposite collecting electrode; F, electrons and scattered photons; G, electron filter, $1.7-\mathrm{mm}$ polystyrene, graphite-coated and connected to ground, positioned $2 \mathrm{~cm}$ from outer grid; $\mathrm{H}$, X-ray beam exit hole, 7.4-cm diam; J, aluminum guard plates, $18 \mathrm{~cm}$ long and $33 \mathrm{~cm}$ high, graphitecoated; K, graphite collecting electrode, $10 \mathrm{~cm}$ by 24 $\mathrm{cm}$ by $5 \mathrm{~mm}$; L, graphite-coated nylon threads; $\mathrm{M}$, vibrating-reed electrometer; $\mathrm{N}$, beam to plate distance, variable from 4 to $22 \mathrm{~cm} ; \mathrm{P}$, distance of beam from opposite wall, variable from 24 to $6 \mathrm{~cm}$; $\mathrm{P}+\mathrm{N}$ is constant at $28 \mathrm{~cm}$ 
The front and back walls of the lead box were fixed in position, as was the X-ray beam, E. The remainder of the box, including the electrode system., was mounted on a track at right angles to the X-ray beam, allowing the variation of the distance, $N$, from the X-ray beam to the collector plate over the range 4 to $22 \mathrm{~cm}$. The distance $P+N$ to the wall opposite the electrode system was $28 \mathrm{~cm}$. That wall and the top and bottom of the box were lined with 1/16-in. graphite-coated Lucite, B, to minimize electron backscattering:

The electron filter, G, consisted of a sheet of graphite-coated polystyrene $1.7 \mathrm{~mm}$ in thickness, electrically connected to ground. When in place, this filter stopped all electrons approaching the collecting volume from outside the filter, but allowed scattered photons to pass through with negligible attenuation. When the filter was removed, both electrons and scattered photons were allowed to pass.

Most of the electrons stopped by the filter originated within the X-ray beam. Hence the difference between the two measurements (filter in, filter out) approximately represents ionization produced by electrons of the roentgen definition. However, the filter also intercepted some electrons produced outside the beam (and outside the filter position) by scattered photons. These electrons were partially replaced by those ejected from the inner surface of the filter itself. As will be seen from the data in figure 4 , such compensation was not complete at $100 \mathrm{kv}$ (or at lower kilovoltages) where photoelectric effect in the air made the filter material slightly non-air-equivalent. It was possible to correct for this, as will be discussed further in relation to figure 4.

Figure 3 is a detail drawing of the grid chamber electrode system, indicating its dimensions and the mounting of the threads. Actually, the grids were made of only one long nylon thread, which was wound around spiral grooves in the two aluminum. rods, F. This thread was then painted with alcohol "dag" in such a way as to leave parts E bare, to serve as insulators, but to connect the inner row of threads to the upper rod and the outer row to the lower rod. External electrical connections were then made to the rods themselves.

The collector plate was made of graphite so that it would be nearly air-equivalent from the standpoint of electron backscattering. To determine the magnitude of this effect, the graphite was replaced with aluminum in one instance for $200-\mathrm{kv}$ X-rays with medium filtration. The ionization measured with the electron filter in place was about 60 percent greater than for the graphite plate because of photoelectric effect in the aluminum. However, the calculated results for electron ionization losses in free-air chambers, based on the aluminum-plate data, did not differ appreciably from those calculated from the graphite-plate data except at small distances from the X-ray beam, as is shown in table 2. Hence since electron scattering varies roughly as $Z^{2}$, one may assume that the experimental results are not significantly influenced by backscatter of electrons from the graphite collector plate.

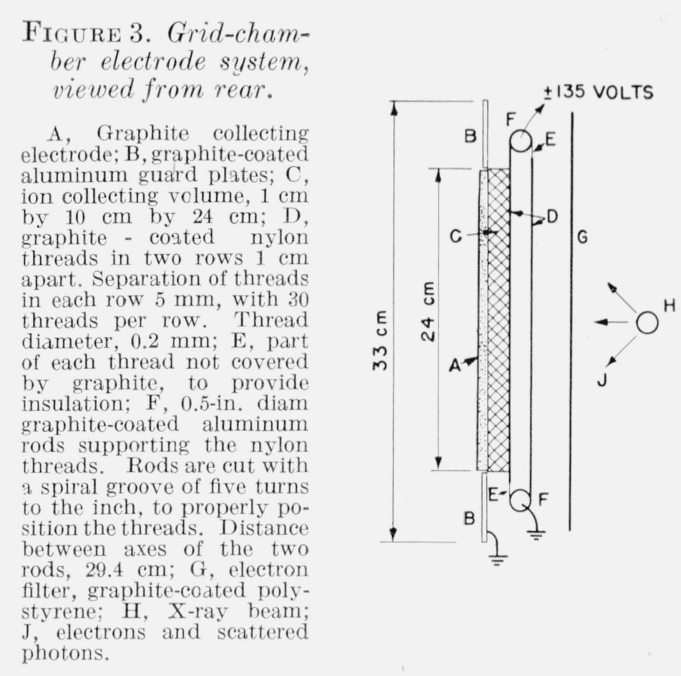

\subsection{Standard Free-Air Chamber}

Measurement of the ionization integrated over the entire ionized volume was made by means of the present NBS standard chamber mentioned in the introduction. Plate separation was $20 \mathrm{~cm}$, plate height, $26.8 \mathrm{~cm}$. The limiting diaphragm diameter $(1 \mathrm{~cm})$ and the collector plate length $(10 \mathrm{~cm})$ were the same as those in the grid chamber. The standard chamber was located on one side of the grid chamber, with the limiting diaphragms of the two chambers in the same plane. The X-ray tube housing was mounted on a track running at right angles to the beam direction, and could thus be positioned in alinement with either chamber. Standard-chamber readings were made in conjunction with each gridchamber run, the X-ray tube output being constant.

One might ask why the standard-chamber reading was necessary, since it would seem. possible to get the total ionization by merely summing up the grid-chamber measurements over the whole volume. This was prohibited by the fact that the gridchamber plate system could not be moved closer to the X-ray beam than about $6 \mathrm{~cm}$ with the electron filcer in place, or $4 \mathrm{~cm}$ without it. Closer positioning would have caused the beam penumbra to strike the grids or filter. Thus, it was not possible with the grid chamber to make measurements in the most densely ionized central region.

Small errors in the standard chamber itself, such as might be caused by slight insufficiency in plate separation, would have a negligible effect on the subsequent calculations of ionization losses.

A detailed description of this standard chamber will be the subject of a later paper.

\subsection{Measurement of Ionization Current}

A vibrating-reed electrometer was used as a nulldetecting device for the current measurements. Currents larger than about $10^{-12}$ amp were passed through calibrated multimegohm resistors, and the resulting IR drops compensated by a potentiometer 
connected in series. For smaller currents a compensating rate-of-charge method was employed, the charge being collected on a calibrated capacitor which was an integral part of the electrometer.

All measurements were carried out at atmospheric pressure and room temperature, approximately 755 $\mathrm{mm} \mathrm{Hg}$ and $26^{\circ} \mathrm{C}$, respectively.

The ionization currents measured in the grid chamber with different polarities of voltage on the inner grid were generally not identical in magnitude, since there was some ionization generated by scattered X-rays in the region behind the collecting electrode. There the grid potential had no influence, but contact-potentials served to collect ionization. This extra-cameral ionization was corrected for by measuring the current with both polarities and averaging the two values. The unwanted current is added in one case and subtracted in the other, and is thus cancelled out.

\subsection{X-Ray Apparatus and Beam Filtration}

A $250 \mathrm{kv}$ X-ray tube enclosed in a thick lead housing was employed for this work. The highvoltage generator supplied an essentially constant potential, having a ripple not exceeding 0.6 percent. Kilovoltage and tube current were continuously monitored during operation and were, by manual adjustment, maintained at a constant value within \pm 0.1 percent.

The inherent filtration of the X-ray tube was equivalent to approximately $3 \mathrm{~mm}$ of $\mathrm{Al}$. Additional filtration ${ }^{4}$ was placed at the 1.27 -cm-diam portal in the tube housing, located $16 \mathrm{~cm}$ from the target. In table 1 are given the added filtrations used at various kilovoltages, together with the copper half-value layers of the filtered beams.

TABLE 1. X-ray beam characteristics: $\quad 7$

\begin{tabular}{|c|c|c|}
\hline $\begin{array}{l}\text { Constant } \\
\text { potential }\end{array}$ & Added a filtration & $\begin{array}{l}\text { Copper half- } \\
\text { value-layers }\end{array}$ \\
\hline \multicolumn{3}{|c|}{ Medium } \\
\hline $\begin{array}{r}k v \\
60 \\
75 \\
100 \\
150 \\
200 \\
250\end{array}$ & $\begin{array}{l}\text { mm } \\
\text { None } \\
1.02 \mathrm{Al}- \\
0.228 \mathrm{Cu}+1.02 \mathrm{Al} \\
0.52 \mathrm{Cu}+1.02 \mathrm{Al} \\
1.02 \mathrm{Cu}+1.02 \mathrm{Al}\end{array}$ & $\begin{array}{l}m m \\
0.077 \\
.099 \\
.176 \\
.60 \\
1.23 \\
2.14\end{array}$ \\
\hline \multicolumn{3}{|c|}{ Heavy } \\
\hline $\begin{array}{l}100 \\
150 \\
200 \\
250\end{array}$ & $\begin{array}{l}0.53 \mathrm{~Pb} \\
\begin{array}{l}1.53 \mathrm{Sn}+4.00 \mathrm{Cu} \\
0.70 \mathrm{~Pb}+4.00 \mathrm{Sn}+0.59 \mathrm{Cu} \\
2.70 \mathrm{~Pb}+1.00 \mathrm{Sn}+0.59 \mathrm{Cu}\end{array}\end{array}$ & $\begin{array}{l}0.77 \\
2.42 \\
4.03 \\
5.28\end{array}$ \\
\hline
\end{tabular}

a Inherent filtration: $3 \mathrm{~mm} \mathrm{Al}$.

The "heavy" filtrations were essentially those of Ehrlich and Fitch [7]; note error in table.4, p. 16, filtration for $150 \mathrm{kv}$ should be $1.53 \mathrm{~mm} \mathrm{Sn}$ and $4 \mathrm{~mm} \mathrm{Cu}$.

\section{Experimental Results}

Figures 4 to 7 are given as examples of the ionization versus distance curves obtained with the grid chamber. Included are curves for $100 \mathrm{kv}$, medium filtration; $200 \mathrm{kv}$, heavy filtration; and $250 \mathrm{kv}$, with both medium and heavy filtration. The filters used were those listed in table 1 . The electron curves have been determined by a point-by-point subtraction of the filter-in curve from the filter-out curve.

The extrapolated range of $100-\mathrm{kev}$ electrons in air at atmospheric pressure is given in the literature [8] as $12 \mathrm{~cm}$. Compton recoil electrons produced by $100-k v$ X-rays will have energies not exceeding 28 $\mathrm{kev}$, and thus ranges less than $2 \mathrm{~cm}$. This identifies the electrons producing the curve in figure 4 as photoelectrons. Therefore, the bend in the upper curve clearly shows the transition from electron ionization to that resulting from scattered X-rays. Beyond $15 \mathrm{~cm}$ the "filter-in" and "filter-out" curves are seen to be parallel and at a constant separation, even though the lower curve has been corrected for attenuation of scattered photons. They should coincide beyond the 12-cm range of the electrons from the X-ray beam. The observed discrepancy is probably caused by the lack of air equivalence of the electron filter material, as was mentioned previously. It is corrected for by raising the lower curve parallel to itself until it coincides with the upper one at large distances.

Figure 5 presenting ionization curves for $200-\mathrm{kv}$ X-rays with heavy filtration also has a sharp bend in the "filter out" curve. However, in this case the "filter-in" curve lies well below it, and thus photon scattering cannot account for the abrupt change in slope. The cause becomes evident if one again considers the types of electrons present. For the X-ray wavelengths present in the primary beam, the absorption coefficient for air will consist of about 2-percent photoelectric effect and 98-percent Compton effect. The range of the maximumenergy photoelectrons will be about $37 \mathrm{~cm}$, whereas the range of the most energetic Compton electrons (88 kev) will be $9 \mathrm{~cm}$. Thus the change in the slope of the curves of figure 5 at $9 \mathrm{~cm}$ represents the transition from Compton electrons to the longer-range photoelectrons.

It should be mentioned in regard to figure 5 (and all other cases of heavy X-ray filtration) that the lower curve, for electron filter in place, had to be estimated in an indirect way. The background current in the grid chamber was of the order of $10^{-15}$ amp and was sometimes unsteady, making it very difficult to obtain reliable measurements of ionization currents of that order of magnitude or smaller. Hence, it was thought preferable to estimate the curve in the following way: The slope was taken in each case to be the same as for the corresponding: medium X-ray filtration as these were found to be about the same for all energies. Then the curve was adjusted to a position such that twice the area under the curve out to $10 \mathrm{~cm}$ was in agreement with 


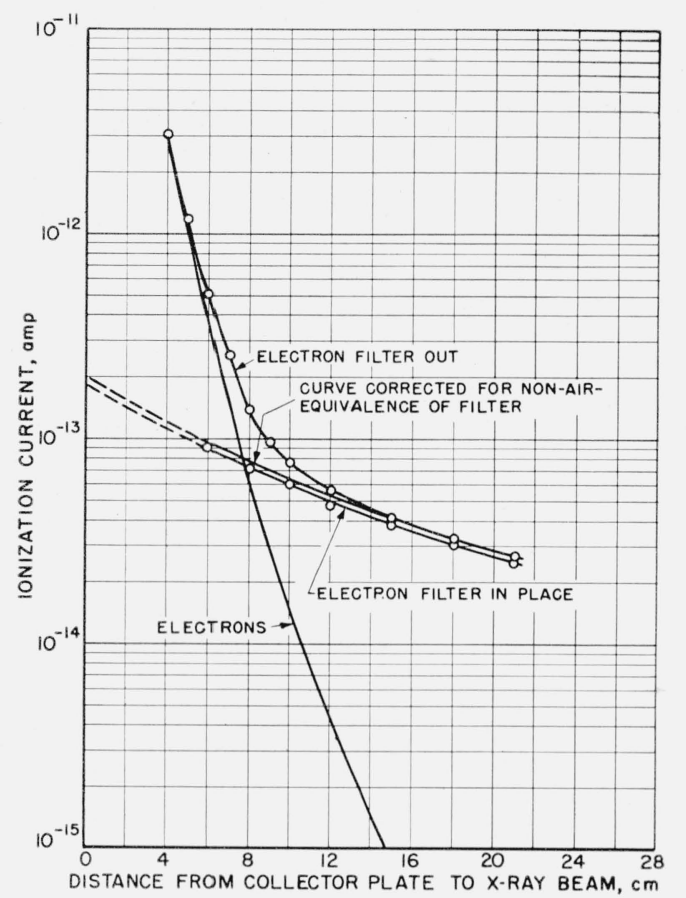

Figure 4. Grid-chamber current versus distance from X-ray beam.

$100 \mathrm{kv}$, medium filtration (1.02-mm Al). Standard free-air chamber current: $4.73 \times 10^{-10} \mathrm{amp}$.

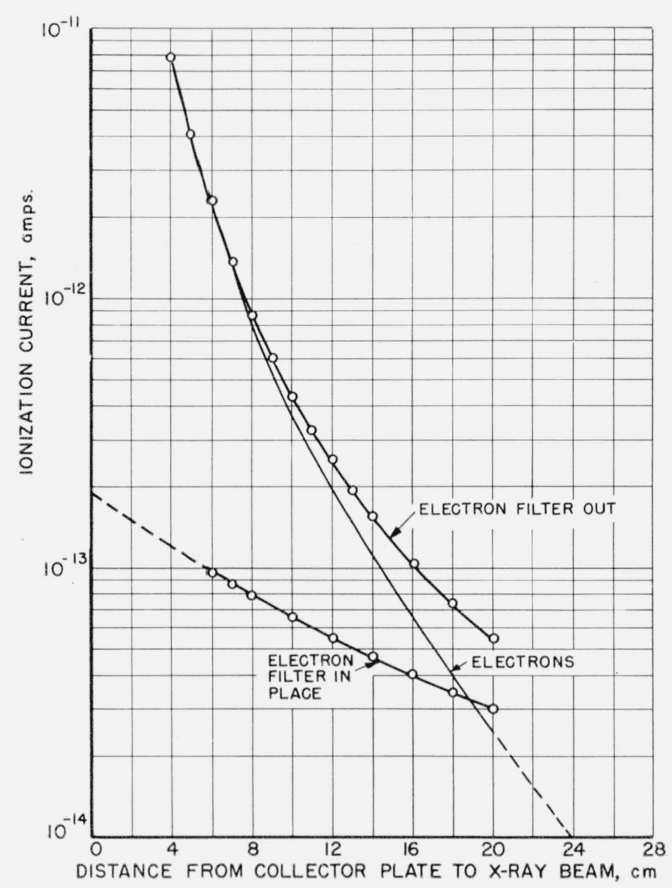

FIGURE 6. Grid-chamber current versus distance from $X$-ray beam.

$250 \mathrm{kv}$, medium filtration $(1.02-\mathrm{mm} \mathrm{Cu}+1.02-\mathrm{mm} \mathrm{Al})$. Standard free-air chamber current: $7.82 \times 10^{-10} \mathrm{amp}$.

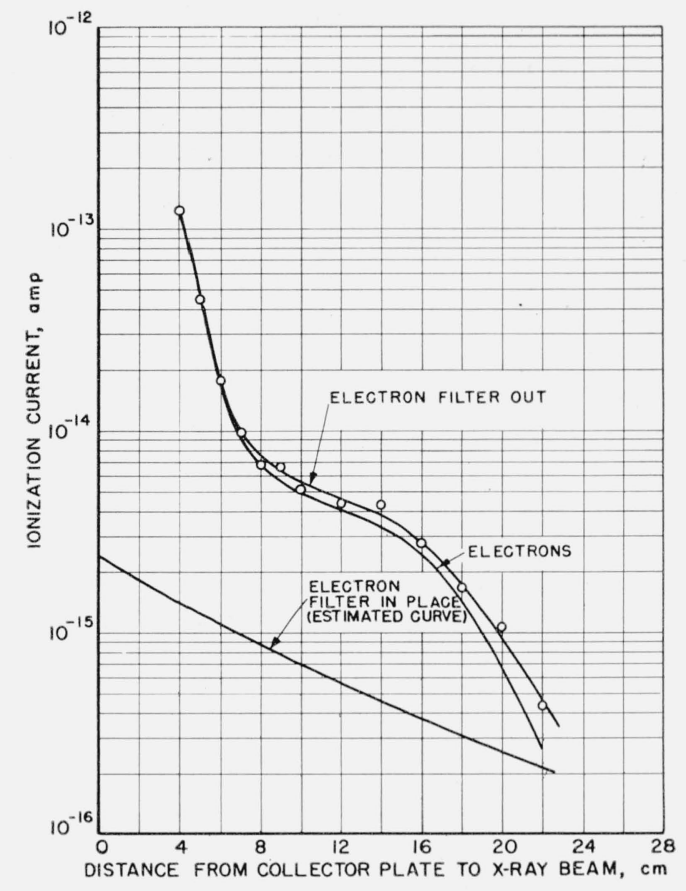

Figure 5. Grid-chamber current versus distance from $X$-ray beam.

$200 \mathrm{kv}$, heavy filtration $(0.70-\mathrm{mm} \mathrm{Pb}+4.00-\mathrm{mm} \mathrm{Sn}+0.59-\mathrm{mm}$ $\mathrm{Cu})$. Standard free-air chamber current: $1.076 \times 10^{-11} \mathrm{amp}$.

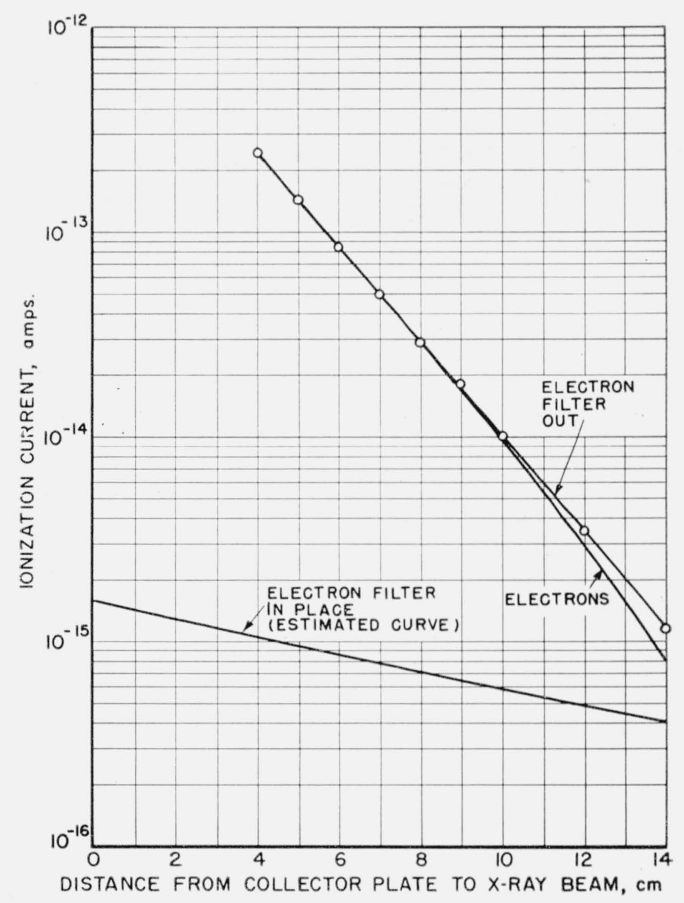

FIGURE 7. Grid-chamber current versus distance from $X$-ray beam.

$250 \mathrm{kv}$, heavy filtration $(2.7-\mathrm{mm} \mathrm{Pb}+1.00-\mathrm{mm} \mathrm{Sn}+0.59-\mathrm{mm}$ $\mathrm{Cu})$. Standard free-air chamber current: $8.89 \times 10^{-12} \mathrm{amp}$. 
the total scattered photon contribution measured in the $20-\mathrm{cm}$ standard free-air chamber, by a method to be described. This was assumed to be proper because a similar comparison between scatter measurements in the free-air chamber and the grid chamber showed generally good agreement for the medium X-ray filtrations.

In figure 7 , representing the ionization curves obtained with heavy filtration at $250 \mathrm{kv}$, the bend observed in figure 5 has disappeared. The photoelectric effect in air is negligible here, and the ionization is almost entirely produced by Compton electrons. Their energies will not exceed $123 \mathrm{kev}$, and hence their ranges extend to only about $15 \mathrm{~cm}$, which appears to be consistent with the curves in figure 7 .

It is thus apparent that the plate-separation requirements for $200 \mathrm{kv}$ with heavy filtration are greater than for $250 \mathrm{kv}$, because of the disappearance of the photoelectric effect in air at the latter energy.

\section{Calculation of Electron Ionization Losses}

For each kilovoltage and filtration the percentage electron-ionization losses caused by insufficient plate separation in free-air chambers were calculated by means of the formula

$$
\frac{(100)(1.087)(2) \sum_{d}^{\infty}\left(I_{e f o}-I_{e f i}\right)}{I_{s c}},
$$

where

$I_{\text {efo }}=$ the grid-chamber ionization current with the electron filter out;

$I_{\text {efi }}=$ the grid-chamber ionization current with the electron filter in place;

$I_{s c}=$ the corresponding ionization current in the standard free-air chamber; $d=$ the variable distance $(\mathrm{cm})$ from collector plate to X-ray beam in the grid chamber. $d-1=s / 2$, where $s$ is the free-air chamber plate separation for which the ionization loss is being computed. 1 is subtracted from $d$ to account for the $1-\mathrm{cm}$ depth of the grid-chamber collecting volume.

The factor (2) is included because the grid chamber measures the ionization on only one side of the X-ray beam. The (1.087) is a correction factor that accounts for the area covered by the nylon threads, which stop the electrons that strike them.

The summation sign indicates that the electron current is summed up, for layers each $1 \mathrm{~cm}$ in thickness, from distance $d$ out to infinity. The electron ionization curves were simply assumed to continue with unchanging slope at plate-to-beam distances exceeding those at which data were obtained.

In figure 8 are plotted the resulting ionization losses for the curves in figures 4 to 7 . Table 2 contains data for all kilovoltages studied.

It should be noted that in the calculations the diameter of the X-ray beam is not considered, and the distances are reckoned from the central axis of the beam. Thus the results are most accurate when applied to chambers having X-ray beams of about the same diameter $(1.8 \mathrm{~cm})$ as was used here.

Thus far, only the losses resulting from inadequate plate separation have been discussed, and no mention has been made of plate height, which is of course of equal importance. A chamber should be designed with both of those dimensions sufficiently large to allow nearly all the electrons projected radially outward from the X-ray beam to run their full ranges without leaving the ion-collecting volume, either by striking the plates at the sides or by escaping out the top and bottom. The data in table 2 represent

TARLe 2. Percentage electron-ionization losses for various plate separations in a free-air chamber

\begin{tabular}{|c|c|c|c|c|c|c|c|c|c|c|c|}
\hline \multirow{3}{*}{ Plate separation } & \multicolumn{11}{|c|}{ Kilovoltage and filtration } \\
\hline & \multirow{2}{*}{$\begin{array}{l}60 \mathrm{kv} \mathrm{Me}- \\
\text { dium }\end{array}$} & \multirow{2}{*}{$\begin{array}{l}75 \mathrm{kv} \text { Me- } \\
\text { dium }\end{array}$} & \multicolumn{2}{|c|}{$100 \mathrm{kV}$} & \multicolumn{2}{|c|}{$150 \mathrm{kv}$} & \multicolumn{3}{|c|}{$200 \mathrm{kv}$} & \multicolumn{2}{|c|}{$250 \mathrm{kv}$} \\
\hline & & & Medium & Heavy & Medium & Heavy & Medium & Medium a & Heavy & Medium & Heavy \\
\hline \begin{tabular}{c}
\multicolumn{1}{c}{$c m$} \\
$6 \ldots \ldots$ \\
$8 \ldots$ \\
10 \\
14 \\
$14 \ldots$
\end{tabular} & $\begin{array}{r}\% \\
0.27 \\
<.05 \\
< \\
-.-1\end{array}$ & $\begin{array}{r}\% \\
0.80 \\
<.20 \\
<.05 \\
-\end{array}$ & $\begin{array}{r}\% \\
2.2 \\
0.8_{1} \\
.32 \\
.13 \\
.0_{6}\end{array}$ & $\begin{array}{r}\% \\
6.4 \\
3.2 \\
1.5 \\
0.67 \\
.28\end{array}$ & $\begin{array}{l}\% \\
4.3 \\
2.1 \\
1.2 \\
0.69 \\
.43\end{array}$ & $\begin{array}{r}\% \\
3.9 \\
3.1 \\
2.5 \\
1.9 \\
1.5\end{array}$ & $\begin{array}{r}\% \\
4.9 \\
2.6 \\
1.6 \\
1.0 \\
0.72\end{array}$ & $\begin{array}{l}\% \\
5.5 \\
2.9 \\
1.8 \\
1.2 \\
0.81\end{array}$ & $\begin{array}{l}\% \\
4.8 \\
2.3 \\
1.4 \\
1.1 \\
0.89\end{array}$ & $\begin{array}{l}\% \\
5.0 \\
2.9 \\
1.8 \\
1.1 \\
0.79\end{array}$ & $\begin{array}{r}\% \\
14.5 \\
8.5 \\
4.9 \\
2.8 \\
1.7\end{array}$ \\
\hline $\begin{array}{l}16 \\
18 \\
20 \\
22 \\
24\end{array}$ & - & p. & $\begin{array}{l}<.05 \\
\ldots \\
-\cdots\end{array}$ & $\begin{array}{r}.13 \\
.07 \\
<.05 \\
\\
\end{array}$ & $\begin{array}{l}.28 \\
.18 \\
.12 \\
.08 \\
.05\end{array}$ & $\begin{array}{l}1.1 \\
0.8_{2} \\
.59 \\
.41 \\
.28\end{array}$ & $\begin{array}{l}.55_{1} \\
.37 \\
.27 \\
.19 \\
.14\end{array}$ & $\begin{array}{l}.59 \\
.43 \\
.33 \\
.25 \\
.19\end{array}$ & $\begin{array}{l}.75 \\
.65 \\
.55 \\
.46 \\
.38\end{array}$ & $\begin{array}{l}.5_{6} \\
.4_{2} \\
.3_{1} \\
.2_{4} \\
.18\end{array}$ & $\begin{array}{l}0.9_{4} \\
.5_{3} \\
.29 \\
.16 \\
.09\end{array}$ \\
\hline $\begin{array}{l}26 \\
28 \\
30 \\
32 \\
34\end{array}$ & $\begin{array}{l}\ldots \\
\cdots \\
\cdots\end{array}$ & מ & p. & 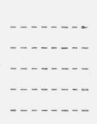 & $\begin{array}{l}<.05 \\
-.-1 \\
-.-.- \\
-.-\end{array}$ & $\begin{array}{r}.19 \\
.12 \\
<.07 \\
<.05 \\
\end{array}$ & $\begin{array}{r}.10 \\
.08 \\
.06 \\
<.05\end{array}$ & $\begin{array}{l}.15 \\
.12 \\
.09 \\
.07 \\
.06\end{array}$ & $\begin{array}{l}.30 \\
.23 \\
.17 \\
.12 \\
.09\end{array}$ & $\begin{array}{l}.1_{4} \\
.1_{1} \\
.09 \\
.07 \\
.06\end{array}$ & \begin{tabular}{c}
$<.05$ \\
\hdashline \\
\hdashline
\end{tabular} \\
\hline 36 & - n...... & - & - n...... & - n-... & -...... & - n-... & - n & $<.05$ & $.0_{6}$ & $<.05$ & - \\
\hline
\end{tabular}

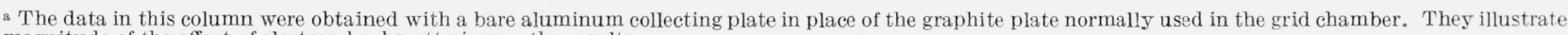
the magnitude of the effect of electron backscattering on the results. 


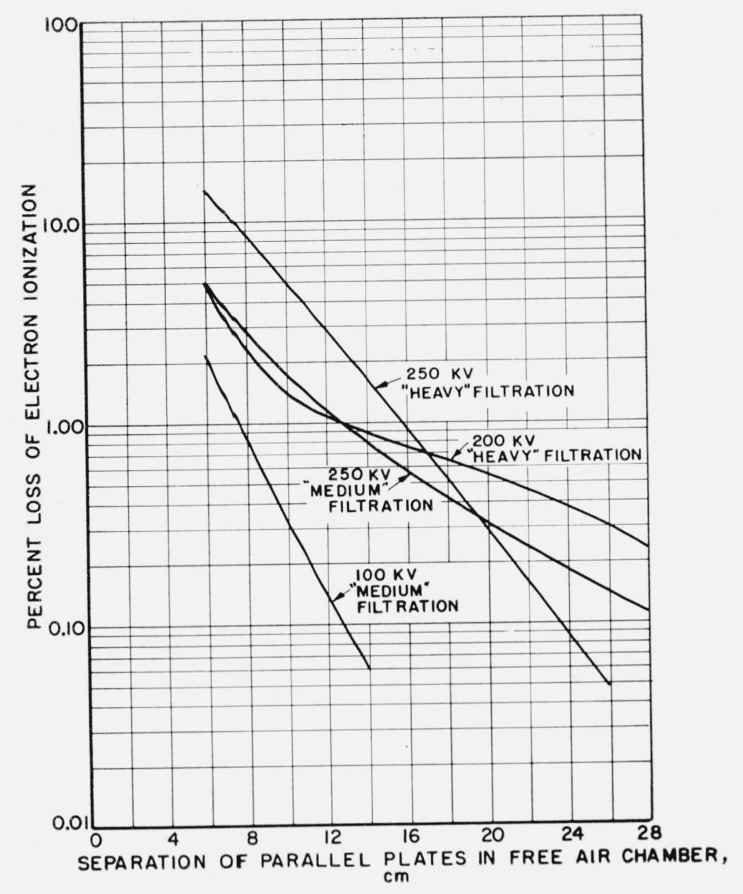

Figure 8. Electron ionization losses in parallelplate free-air chambers with adequate plate height.

losses caused by plate-separation inadequacy alone, assuming the plate height to be adequate. What constitutes adequate plate height is complicated by field distortion, as pointed out by Kemp and Hall [5]. The collecting volume may be defined as the region occupied by the electric-field lines that terminate on the collector plate. This region will be considerably smaller in effective height than the plates themselves, if the field is not properly guarded from the effects of the grounded chamber enclosure. The actual cross-sectional shape of the collecting volume of a particular chamber, in a plane at right angles to the X-ray beam, is obtainable from a study of field lines in an electrolytic tank.

It was thought to be desirable to convert the data in table 2 into a more general form that could be applied to any free-air chamber in which the crosssectional shape of the collecting volume were known. A graphical method was found for doing this.

The graph used in this calculation was similar to that shown in figure 9 , but drawn to a large scale. The ionization field was assumed to be made up of concentric cylindrical shells, each $1 \mathrm{~cm}$ in thickness and containing a uniform ionization density. The superimposed rectangles, $24 \mathrm{~cm}$ in height and $1 \mathrm{~cm}$ in thickness, represent various positions of the gridchamber collecting volume. By first taking planimeter measurements, the unknown ionization densities in the cylindrical shells were deduced from the known electron ionizations in the grid-chamber volume. It was then possible to calculate how much of the ionization lay outside of any given radius, i. e., the ionization that would be lost by a cylindrical chamber of that radius, positioned coaxially with the beam. Table 3 gives the results of such calculations.

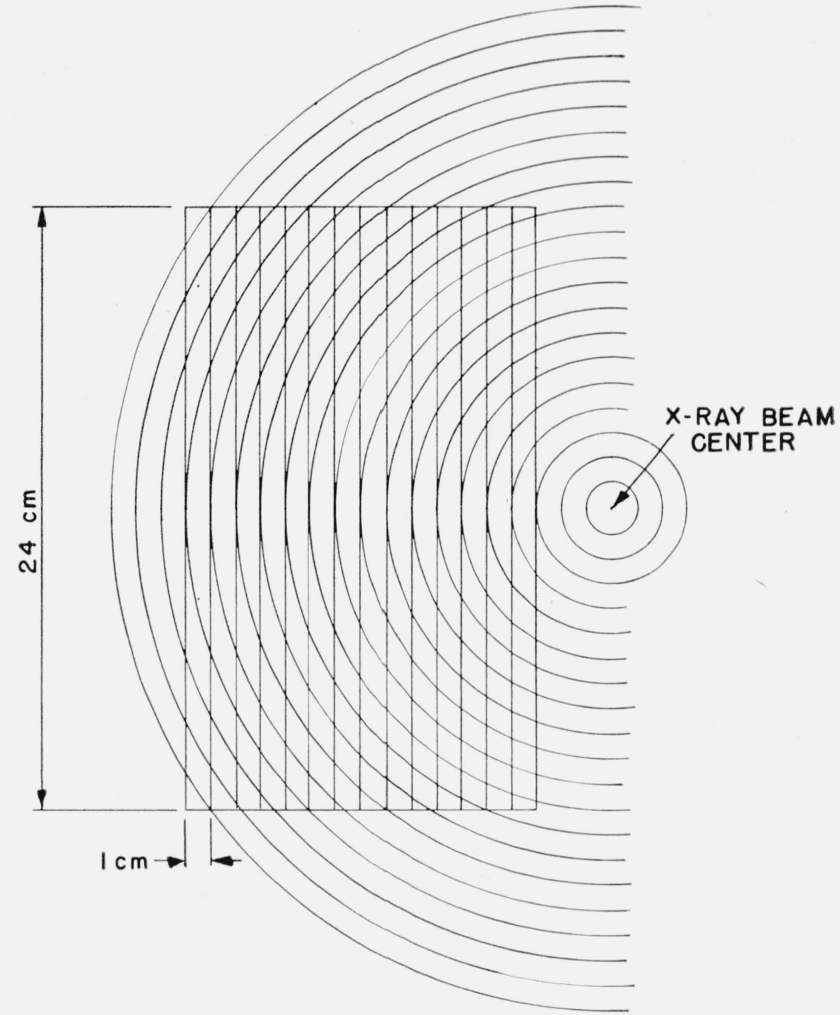

Figure 9. Drawing similar to that used for planimeter measurements to determine the ionization densities existing in cylindrical shells coaxial with the $X$-ray beam.

To apply these results to any chamber for which the cross-sectional shape of the collecting volume is known, it is necessary to plot the cross section on polar-coordinate graph paper, with the X-ray beam located at the origin. The ionization losses are then obtained from table 3 for a number of representative radii on the plot, weighted according to the angular sector they represent, and summed to give the total ionization loss.

A calculation of this sort was carried out to determine the adequacy of the 24-cm height of the collector plate in the grid chamber, and the effect on the data in table 2 . In the worst cases $(250 \mathrm{kv}$, medium filtration; $200 \mathrm{kv}$, heavy filtration) the tabulated percentage losses in table 2 were found to be too small by about 0.2 percent for figures of 1 percent or larger, and by 0.1 percent or less for figures below 1 percent. The data in table 3 are not subject to this error as the actual collector-plate height of the grid chamber was taken into account in the calculation of that data.

The ionization contributed by scattered X-rays in a particular free-air chamber will depend not only upon the plate separation and height, but also upon the length of the irradiated air column within the chamber enclosure; thus, this ionization cannot be conveniently tabulated. It was therefore considered preferable to describe a method by which the ioniza- 
TABLE 3. Percentage losses of electron ionization outside of various radii from the X-ray beam

\begin{tabular}{|c|c|c|c|c|c|c|c|c|c|c|}
\hline \multirow{3}{*}{ Radius } & \multicolumn{10}{|c|}{ Kilovoltage and filtration } \\
\hline & \multirow{2}{*}{$\begin{array}{l}60 \mathrm{kv} \mathrm{Me}- \\
\text { dium }\end{array}$} & \multirow{2}{*}{$\begin{array}{l}75 \mathrm{kv} M e- \\
\text { dium }\end{array}$} & \multicolumn{2}{|c|}{$100 \mathrm{kV}$} & \multicolumn{2}{|c|}{$150 \mathrm{kv}$} & \multicolumn{2}{|c|}{$200 \mathrm{kV}$} & \multicolumn{2}{|c|}{$250 \mathrm{kV}$} \\
\hline & & & Medium & Heavy & Medium & Heavy & Medium & Heavy & Medium & Heavy \\
\hline $\mathrm{cm}$ & $\%$ & $\%$ & $\%$ & $\%$ & $\%$ & $\%$ & $\%$ & $\%$ & $\%$ & $\%$ \\
\hline $\begin{array}{l}3 \\
4 \\
5 \\
6 \\
7\end{array}$ & $\begin{array}{r}0.77 \\
.10 \\
<.05 \\
\\
\end{array}$ & $\begin{array}{r}2.1 \\
.5_{6} \\
.1_{5} \\
<.05 \\
\end{array}$ & $\begin{array}{l}4.9 \\
2.0 \\
.88 \\
.37 \\
.17\end{array}$ & $\begin{array}{r}12.9 \\
7.6 \\
\text { 4. } 1 \\
2.0 \\
.87\end{array}$ & $\begin{array}{l}\text { 8. } 9 \\
\text { 4. } 7 \\
\text { 2. } 7 \\
\text { 1. } 65 \\
\text { 1. } 1\end{array}$ & $\begin{array}{l}6.0 \\
5.3 \\
4.6 \\
3.9 \\
3.3\end{array}$ & $\begin{array}{l}9.7 \\
5.5 \\
3.5 \\
2.3 \\
1.7\end{array}$ & $\begin{array}{r}10.4 \\
5.0 \\
2.9 \\
2.2 \\
1.8\end{array}$ & $\begin{array}{l}9.9 \\
6.1 \\
4.0 \\
2.7 \\
1.9\end{array}$ & $\begin{array}{r}27.7 \\
18.1 \\
11.5 \\
7.2 \\
4.4\end{array}$ \\
\hline 10 & (n- & (n- & \begin{tabular}{l}
.09 \\
$<.05$ \\
\hdashline $.0-$ \\
$-1 .+\cdots$
\end{tabular} & $\begin{array}{l}38 \\
.20 \\
11_{2} \\
08 \\
.05\end{array}$ & $\begin{array}{l}.74 \\
.5_{1} \\
.35 \\
.24 \\
.17\end{array}$ & $\begin{array}{l}\text { 2. } 65 \\
2.1 \\
1.6 \\
1.2 \\
.88\end{array}$ & $\begin{array}{l}1.3 \\
.9_{4} \\
.7_{2} \\
.5_{5} \\
.42\end{array}$ & $\begin{array}{l}1.6 \\
1.4 \\
1.3 \\
1.1 \\
1.0\end{array}$ & $\begin{array}{l}1.4 \\
1.1 \\
.8_{6} \\
.68 \\
.55\end{array}$ & $\begin{array}{l}2.7 \\
1.6 \\
.97 \\
.5_{5} \\
.3_{0}\end{array}$ \\
\hline 1314 & (n) & - & (n- & $<.05$ & $\begin{array}{r}.12 \\
.08 \\
.06 \\
<.05 \\
\end{array}$ & $\begin{array}{l}.63 \\
.43 \\
.28 \\
.18 \\
.10\end{array}$ & $\begin{array}{l}.3_{2} \\
.24 \\
.1_{8} \\
.1_{4} \\
.1_{1}\end{array}$ & $\begin{array}{l}.87 \\
.7_{2} \\
.57 \\
.44 \\
.32\end{array}$ & $\begin{array}{l}.44 \\
.3_{6} \\
.30 \\
.25 \\
.20\end{array}$ & $\begin{array}{r}.16 \\
.06 \\
<.05 \\
\\
\end{array}$ \\
\hline $\begin{array}{l}18 \\
19 \\
20 \\
21 \\
22\end{array}$ & (n- & (n-1 & - & - n & (n- & $<.05$ & $\begin{array}{r}.08 \\
.06 \\
<.05 \\
\\
\end{array}$ & $\begin{array}{r}.23 \\
.15 \\
.10 \\
.06 \\
<.05\end{array}$ & $\begin{array}{l}.17 \\
.14 \\
.12 \\
.10 \\
.09\end{array}$ & - \\
\hline
\end{tabular}

tion by scattered X-rays can be directly determined with sufficient accuracy in any given chamber.

A tube of nearly air-equivalent material such as Lucite, extending the full length of the chamber enclosure, is positioned inside the chamber, so that the X-ray beam passes through it from end to end. The tube must have walls thick enough to stop the electrons, but thin with respect to attenuation of scattered X-rays. The plastic should be made conducting with a graphite coating, and operated at half the potential of the high voltage plate, to minimize field distortion. The X-ray beam is narrowly collimated to prohibit the penumbra from producing excess scatter by striking the walls of the tube, which should be as small in diameter as practicable. The ratio of ionization measured with the tube in place to that with it removed will give the fraction of the total ionization that is contributed by scattered $\mathrm{X}$-rays. As most of the electrons have short ranges (see table 3), the normal distribution of electrons generated by scattered X-rays in the chamber will not be significantly disturbed by the presence of the tube. A small error will be produced by the "shadowing" effect of the tube because it collects the ionization produced between it and the highvoltage plate, prohibiting that ionization from arriving at the collector plate. This can be corrected with sufficient accuracy by simply taking into account the collecting volume lost by the effect. Details of the application of this method to the NBS standard chamber will be included in a later paper; however, the ionization from scattered X-rays in that chamber was found to constitute 0.3 to 0.4 percent of the total ionization, increasing slowly with decreasing energy.

The net ionization loss of a free-air chamber is found by subtracting the measured contribution due to scattered X-rays from the appropriate figures for loss of electron ionization as given in tables 2 and 3 .

\section{Comparison of Grid-Chamber Results With Other Measurements}

Two chambers were constructed whose predicted ionization losses (from grid-chamber results) were large because of inadequate dimensions. Care was taken to avoid field distortion in these chambers. The actual deficiency was then determined experimentally for 200-kv X-rays with medium filtration by comparison with the $20-\mathrm{cm}$ standard chamber. In the latter chamber electron losses and scattered photon contributions compensate one another within about 0.05 percent at that energy.

Table 4 gives the results for a chamber of 24-cm height and separations from 6 to $9 \mathrm{~cm}$. Table 5 gives similar data for a chamber $10 \mathrm{~cm}$ in height and the same range of separations. Agreement with grid-chamber predictions is good in both cases.

In the light of these results and other considerations, the limits of error of the electron-loss data in tables 2 and 3 are estimated to be \pm 0.3 percent, except for the grid-chamber plate-height inadequacy, which affects only table 2 . The loss figures are

TABLE 4. Comparison of predicted and measured deficiencies for parallel plate chambers

$24 \mathrm{~cm}$ plate height

\begin{tabular}{|c|c|c|}
\hline $\begin{array}{c}\text { Free-air } \\
\text { chamber } \\
\text { plate sepa- } \\
\text { ration }\end{array}$ & $\begin{array}{c}\text { Measured } \\
\text { ionization } \\
\text { deficiency }\end{array}$ & $\begin{array}{c}\text { Ionization a } \\
\text { deficiency } \\
\text { from grid- } \\
\text { chamber } \\
\text { data }\end{array}$ \\
\cline { 1 - 1 }$c m$ & $\%$ & $\%$ \\
6 & 4.9 & 5.0 \\
7 & 3.5 & 3.6 \\
8 & 2.7 & 2.7 \\
9 & 2.1 & 2.1 \\
\hline
\end{tabular}

a These figures have been raised by 0.1 percent to account for the estimated difference in scattered photon ionization in the $20-\mathrm{cm}$ standard chamber and the test chamber. 
TABLE 5. Comparison of predicted and measured deficiencies for parallel-plate chambers

$10-\mathrm{cm}$ plate height

\begin{tabular}{|c|c|c|}
\hline $\begin{array}{c}\text { Free-air } \\
\text { chamber } \\
\text { plate sepa- } \\
\text { ration }\end{array}$ & $\begin{array}{c}\text { Measured } \\
\text { ionization } \\
\text { deficiency }\end{array}$ & $\begin{array}{c}\text { Ionization a } \\
\text { deficiency } \\
\text { from grid- } \\
\text { chamber } \\
\text { data }\end{array}$ \\
\cline { 1 - 1 }$c m$ & $\%$ & $\%$ \\
6 & 6.0 & 5.8 \\
7 & 4.7 & 4.7 \\
8 & 3.9 & 3.9 \\
9 & 3.3 & 3.3 \\
\hline
\end{tabular}

a These figures have been raised by 0.15 percent for the reason noted in table 4

probably within closer limits of error at larger plate separations, where the losses themselves are decreased.

\subsection{Comparison With Results of Kemp and Hall}

The results of the measurements of Kemp and Hall [5] are expressed as ionization losses in chambers having square cross sections of various sizes. These ionization loss figures are compared with corresponding figures from the present work in table 6, for $100-\mathrm{kv}$ X-rays.

TABLE 6. Comparison with work of Kemp and Hall

\begin{tabular}{|c|c|c|}
\hline $\begin{array}{c}\text { Square-chamber } \\
\text { dimensions }\end{array}$ & $\begin{array}{c}\text { Ionization losses of } \\
\text { Kemp and Hall, } \\
100 \mathrm{kvp}\end{array}$ & $\begin{array}{c}\text { Electron - ionization } \\
\text { losses from grid- } \\
\text { chamber measure- } \\
\text { ments, 100-kv con- } \\
\text { stant potential }\end{array}$ \\
\hline$c m$ & $\% \%$ & $\%$ \\
$9.6 \times 9.6$ & $<0.1$ & 0.7 \\
$7.4 \times 7.4$ & 1.5 & 1.9 \\
$6.5 \times 6.5$ & & 3.0 \\
\hline
\end{tabular}

It is evident that large differences exist between the two sets of data, the ionization losses of Kemp and Hall being smaller than reported here. Similar discrepancies were found to exist at the other kilovoltages as well. The most important factors contributing to these differences are:

1. Difference in quality of the X-rays used. The copper half-value-layer obtained by Kemp and Hall at $100 \mathrm{kvp}$ was $0.078 \mathrm{~mm},{ }^{5}$ as compared with a value of $0.176 \mathrm{~mm}$ obtained in the present experiment. Kemp and Hall used a Villard circuit and 2-mm Al inherent filtration, compared with a constant potential generator and 3-mm Al inherent filtration.

2. Difference in X-ray beam diameter. Kemp and Hall employed a beam about $1 \mathrm{~cm}$ in diameter, as compared with the present $1.8 \mathrm{~cm}$.

\subsection{Comparisons With Work of Others}

To facilitate comparisons with other experiments at kilovoltages diftering from those used for the present experiment, a graph of plate separation versus kilovoltage is given in figure 10 for medium

\footnotetext{
5 Figure supplied by Kemp in a private communication.
}

X-rày filtration. Also plotted on this graph are the points at which complete ${ }^{6}$ collection of ionization was observed by other investigators. Constantpotential kilovoltages were used in every case. Filtrations were not given except by Duane and Lorenz, who used $2-\mathrm{mm}$ Al at $95 \mathrm{kv}$, but did not state the filtrations for other kilovoltages, and by Failla, who used $0.1-\mathrm{mm} \mathrm{Cu}$ and $2.15-\mathrm{mm} \mathrm{Al}$ at $110 \mathrm{kv}$. One may assume a medium or light filtration was employed in all the other instances.

The results of Taylor, Singer, and Charlton [9] are essentially in agreement with the present gridchamber data, since it is unlikely that they could have detected by their method the very small increments of ionization existing at greater separations. Their ionization chamber was relatively free from electricfield distortion at separations up to $18 \mathrm{~cm}$, because of the long guard plates $(25 \mathrm{~cm})$, the large collectingplate height $(35 \mathrm{~cm})$, and a system of guard wires, all enclosed in a tank of ample dimensions $(65-\mathrm{cm}$ diam by 213-cm length). The other data of Taylor [10] were given as part of a general survey paper, and were not all obtained with an individual chamber. The point at $240 \mathrm{kv}$ was taken from reference [9]. The other data were taken in two other chambers, each of which is now known, in the light of recent developments, to have been subject to considerable field distortion, accounting for the low values obtained for the separations.

The same explanation also applies to the results of Kaye and Binks [11], Duane and Lorenz [12], and Failla [13].

Failla's work was particularly interesting, inasmuch as he made a careful study of the field distor-

${ }^{6}$ The other investigators used the method of varying the plate separation while observing the ionization current. Complete ionization collection was assumed to exist at the point where no detectable increase in ionization was observed with further increase in separation. Their methods did not differentiate between electron ionization and that produced by scattered X-rays, but simply measured the total of the two.

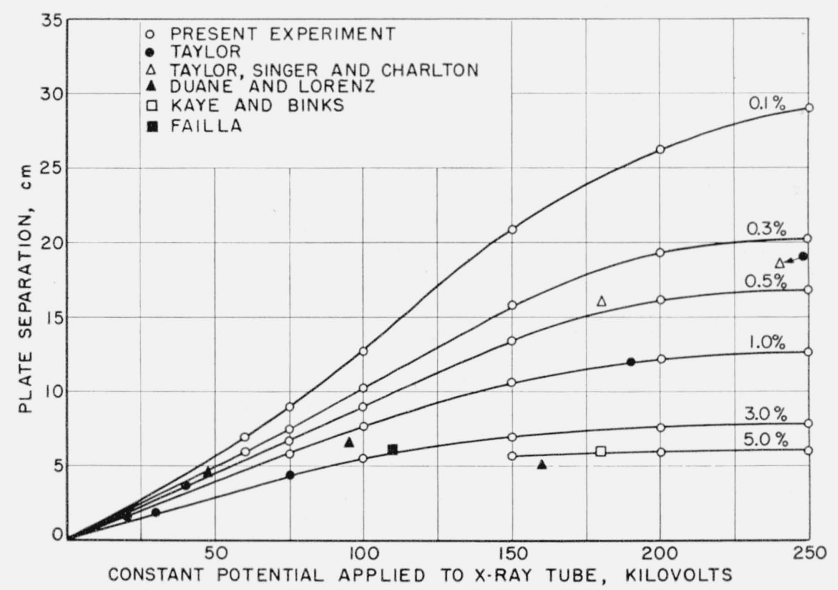

Figure 10. Comparison with results of other workers for medium X-ray filtrations and constant potentials.

The curves are drawn through points obtained in the present experiment, each curve indicating the plate separations at which equal losses of electron ionization occur for various kilovoltages. The other points represent separations at which occur for various kilovoltages. The other points represent separations at
other workers observed complete collection of ionization (see footnote 6 ). 
tion existing within his chamber. His collecting-plate was divided into 11 adjacent vertical strips, each 3.8 $\mathrm{cm}$ wide, so that he could measure the ionization in various volumes along the X-ray beam. He found that the ionization currents collected by several of the centrally located strips were the same, and that the closer the plate separation, the larger the number of adjacent strips collecting the same current. This was interpreted as an indication of the region over which no field distortion was present. One could equally well interpret the same facts as showing the region over which the distortion produced the same error in each strip. The latter would seem to be the case, since, in another measurement, Failla varied the plate separation from 6 to $20 \mathrm{~cm}$ and measured a constant current output from the central strip out to $15-\mathrm{cm}$ separation. The present work shows that the current should have increased by about 3 percent over this range of separations, for $110-\mathrm{kv}$ X-rays with medium filtration. Hence, the effective collecting volume of the central strip probably decreased by about 3 percent because of worsening field distortion. This has been substantiated by preliminary measurements of field distortion by Miller and Kennedy at the Bureau.

The field-distortion problem is thus seen to be much more serious than has been heretofore supposed, and new criteria must be established for designing distortion-free chambers. This problem is now under investigation.
The authors express their appreciation to H. O. Wyckoff for many helpful discussions relating to this work.

\section{References}

[1] L. S. Taylor, BS J. Research 8, 9 (1932) RP397; Radiology 18, 99 (1932)

[2] L. S. Taylor, BS J. Research 8, 325 (1932) RP417; Radiology 18, 627 (1932).

[3] L. S. Taylor and G. Singer, BS J. Research 5, 507 (1930) RP211; Radiology 15, 637 (1930)。

[4] L. S. Taylor and G. Singer, J. Research NBS 16, 165 (1936) RP865; Radiology $\mathbf{2 6 ,} 322$ (1936).

[5] L. A. W. Kemp and S. M. Hall, Brit. J. Radiol. 27, 219 (1954).

[6] H. O. Wyckoff, G. H. Aston, and E. E. Smith, Brit. J. Radiol. 27, 325 (1954).

[7] M. Ehrlich and S. H. Fitch, Nucleonics 9, 5 (1951).

[8] N. N. Das Gupta and S. H. Ghosh, Revs. Mod. Phys. 18, $280(1946)$.

[9] L. S. Taylor, G. Singer, and A. L. Charlton, J. Research NBS 21, 19 (1938) RP1111.

[10] L. S. Taylor, Brit. J. Radiol. 24, 67 (1951).

[11] G. W. C. Kaye and W. Binks, Brit. J. Radiol. 6, 530 (1933).

[12] W. Duane and E. Lorenz, Am. J. Roentgenol. 19, 461 (1928).

[13] G. Failla, Am. J. Roentgenol. 21, 47 (1929).

WAshington, July 8, 1954. 